\section{Hipertensão pulmonar em pacientes com hipertrofia adenoamigdaliana}

\author{
Bruno Bartolomei Sebusiani ${ }^{1}$, Shirley Pignatari ${ }^{2}$, \\ Giuseppe Armínio ${ }^{3}$, Levon Mekhitarian Neto ${ }^{4}$, \\ Aldo E. Cassol Stamm 5
}

\section{Resumo / Summary}

$\Delta$ esar de a hipertrofia adenoamigdaliana ser uma moléstia reconhecida desde os primórdios, foi somente descrita como causadora de hipertensão pulmonar e cor pulmonale em 1965 por Menashe e Farrehi. Ainda são poucos os casos apresentados na literatura analisando a existência de hipertensão pulmonar em crianças com aumento do tecido adenoamigdaliano. Objetivo: Avaliar a prevalência de hipertensão pulmonar em crianças com hipertrofia adenoamigdaliana. Forma de estur do: Coorte transversal. Material e Método: Foram avaliados 14 pacientes, com idade inferior a 15 anos, de ambos os sexos, com indicação formal para adenoamigdalectomia por hipertrofia adenoamigdaliana. Todos os pacientes foram submetidos à avaliação pré-operatória contendo: questionário, avaliação clínica otorrinolaringológica, Rx simples de cavum, vídeo-nasofibroscopia e ecocardiograma. Resultados: Dos 14 pacientes analisados, um $(7,14 \%)$ apresentou hipertensão pulmonar. Conclusão: Com base em nosso estudo, concluímos que a hipertrofia adenoamigdaliana está relacionada com o desenvolvimento de hipertensão pulmonar, sendo o ecocardiograma um exame bastante útil na determinação desta condição clínica. A remoção cirúrgica do tecido adenoamigdaliano pode reverter o quadro de hipertensão pulmonar decorrente do processo obstrutivo crônico.

\section{Pulmonary hipertension in patients with adenotonsillar hypertrophy}

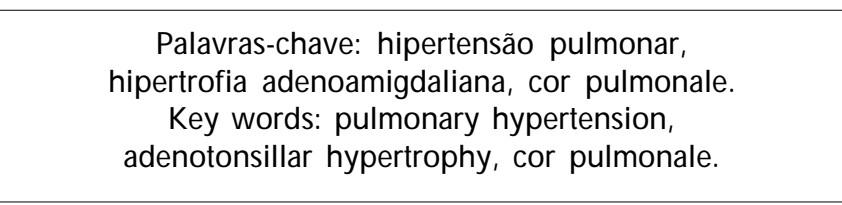

\begin{abstract}
A
Ithough adenotonsillar hypertrophy is a well-recognized disease since the beginning, it was not described to be cause of pulmonary hypertension until 1965 by Menashe and Ferrehi. There are only few records in the literature, analyzing the existence of pulmonary hypertension in children with obstructive adenotonsillar hypertrophy. Aim: The aim of this study was to evaluate the prevalence of pulmonary hypertension in children with obstructive adenotonsillar hypertrophy. Study design: Transversal cohorte. Material and Method: Fourteen patients with indication for adenotonsillectomy, from both sexs, with age ranging from 0 to 15 years, were analyzed in this study. All patients underwent to a complete pre-surgery evaluation, with questionary, complete ENT evaluation, lateral $X$ ray films, nasofibrolaryngoscopy and echocardiography. Results: From all fourteen patients analyzed, one $(7,14 \%)$ had pulmonary hypertension in the echocardiography examination. Conclusion: Adenotonsillar hypertrophy is a cause of pulmonary hypertension, and echocardiography examination is a very usefull exam to determine to presence of the pathology. Adenotonsillectomy may revert pulmonary hypertension in children with adenotonsillar hypertrophy.
\end{abstract}

\footnotetext{
${ }^{1}$ Residente do terceiro ano de Otorrinolaringologia do Hospital Prof. Edmundo Vasconcelos - Centro de O torrinolaringologia e Fonoaudiologia de São Paulo. ${ }^{2}$ Otorrinolaringologista responsável pelo setor de O torrinolaringologia Pediátrica do Hosp. Prof. Edmundo Vasconcelos - Centro de Otorrinolaringologia e Fonoaudiologia de São Paulo; Professora Adjunta da Disciplina de Otorrinolaringologia Pediátrica da Universidade Federal de São Paulo. ${ }^{3}$ Cardiologista responsável pelo setor de Ecocardiografia do Hosp. Prof. Edmundo Vasconcelos.

${ }^{4}$ Otorrinolaringologista do Hosp. Prof. Edmundo Vasconcelos - Centro de O torrinolaringologia e Fonoaudiologia de São Paulo; pós-graduando em Cirurgia de Cabeça e Pescoço do Hospital Heliópolis.

${ }^{5}$ Diretor do setor de O torrinolaringologia do Hosp. Prof. Edmundo Vasconcelos - Centro de O torrinolaringologia e Fonoaudiologia de São Paulo. Endereço para Correspondência: Centro de Otorrinolaringologia e Fonoaudiologia de São Paulo Hospital Prof. Edmundo Vasconcelos - Rua Borges Lagoa, 1450 Vila Clementino 04038-905 São Paulo SP. Tel (0xx11) 5080-4357 - E-mail: centrodeorl@osite.com.br

Artigo recebido em 11 de agosto de 2003. Artigo aceito em 04 de setembro de 2003.
} 


\section{INTRODUÇÃO}

Todo médico que dedica seu tempo para o atendimento de crianças já se questionou sobre as razões pelas quais são indicadas as adenoamigdalecto mias. Várias complicações são sabidamente resultado direto da obstrução causada pela hipertrofia adenoamigdaliana, como por exemplo: respiração bucal de suplência, alterações no desenvolvimento crânio-facial, apnéia obstrutiva do sono, dificuldade de alimentação, entre outros, sendo todas as condições supracitadas possíveis indicações cirúrgicas. Entre as complicações mais graves decorrentes do processo obstrutivo crônico decorrente do aumento exagerado das vegetações adenoideanas e amígdalas palatinas, incluem-se a hipertensão pulmonar e cor pulmonale. Embora não se saiba com exatidão sua prevalência, esta condição clínica é considerada uma indicação incontestável para a realização da adenoamigdalectomia.

Registros hindus de cerca de 3.000 anos relatam um ato cirúrgico que seria posteriormente descrito como amigdalectomia. Este procedimento envolvia o descolamento da cápsula mucosa com o dedo e a enucleação da amídala palatina. Celsius e Paulo de Algena também realizavam procedimento semelhante há aproximadamente 2.000 anos. Acredita-se que a primeira adenoidectomia foi realizada no fim do século XIX, quando Wilhem Meyer de Copenhagem sugeriu que as vegetações adenóides seriam responsáveis pela deficiência auditiva assim como pelos sintomas nasais apresentados por inúmeros pacientes.

Sendo a adenoamidalectomia um procedimento com pequena morbi-mortalidade, houve um grande entusiasmo pela realização da cirurgia, principalmente na primeira metade do século passado. Esta era, muitas vezes, indicada para pacientes com sintomatologia mínima ou portadores de moléstias não relacionadas às amígdalas palatinas e vegetações adenoideanas. Na década de 1970, nos EUA, foram realizadas proporcionalmente 1.200 adenoamigdalectomias para cada 100.000 intervenções cirúrgicas gerais ${ }^{1}$. As décadas seguintes, no entanto, foram marcadas por um ceticismo quanto à indicação da adenoamigdalectomia, observando-se uma redução de aproximadamente $30 \%$ no número de cirurgias realizadas!

A pesar desta diminuição significativa, a adenoamigdalectomia ainda é o procedimento cirúrgico mais realizado na faixa etária pediátrical. A obstrução crônica das vias aéreas, seguida de hipertensão pulmonar e cor pulmonale, é uma indicação incontestável para a realização da adenoamigdalectomia.

0 objetivo deste estudo foi avaliar a prevalência de hipertensão pulmonar em pacientes com hipertrofia adenoamigdaliana.

\section{MATERIAL E MÉTODO}

Este estudo prospectivo foi realizado nos Departa- mentos de Otorrinolaringologia (Otorrinopediatria) e Ecocardiografia do Hospital Professor Edmundo Vasconcelos, no período de março a outubro de 2002. Foram incluídos 14 pacientes, com idade inferior a 15 anos, de ambos sexos, com indicação formal para adenoamigdalectomia por hipertrofia adenoamigdaliana. Foram excluídos do presente estudo pacientes com má-formação crânio-encefálica, síndromes genéticas, discrasias sanguíneas, e outras indicações para adenoamigdalectomia que não a hipertrofia adenoamigdaliana. Todos os pacientes tiveram o termo de consentimento escrito em relação aos procedimentos clínicocirúrgicos assinado por um maior responsável.

Todos os pacientes foram submetidos à avaliação préoperatória contendo: questionário, avaliação clínica otorrinolaringológica, Rx simples de cavum, vídeonasofibroscopia e ecocardiograma.

Um questionário contendo perguntas relacionadas às queixas obstrutivas apresentadas pelo paciente foi respondido por um maior responsável durante o préoperatório, objetivando estimar o impacto da hipertrofia adenoamigdaliana na qualidade de vida do paciente. Em caso de positividade da queixa, esta era, posteriormente, classificada em relação à sua gravidade (pouca, intermediária ou elevada).

Durante o exame otorrinolaringológico, pôde-se avaliar o tamanho das amígdalas palatinas. As amígdalas foram, então, classificadas em 4 diferentes graus, dependendo do grau de obstrução proporcionado ao nível da orofaringe. Convencionou- se como grau I as obstruções amigdalianas de até $25 \%$ da orofaringe; grau II, obstrução de $25-50 \%$; grau III, obstrução de $50-75 \%$ e grau IV, obstrução maior que $75 \%$ da luz da orofaringe.

A avaliação da hipertrofia adenoideana foi avaliada tanto pelo Rx simples de cavum quanto pela vídeo-nasofibroscopia. Estas variáveis também foram classificadas em quatro diferentes níveis, dependendo da obstrução evidenciada ao exame. Os mesmos critérios utilizados para se avaliar o grau de obstrução produzido pela hipertrofia amigdaliana foram empregados para a avaliação da obstrução promovida pelo aumento do tecido adenoideano. 0 grau de hipertrofia adenoideana evidenciado à vídeo-nasofibroscopia foi posteriormente correlacionado à obstrução proporcionada pela vegetação adenoideana ao Rx simples de cavum. A vídeo-nasofibroscopia foi realizada com endoscópio flexível (Machida $3.2 \mathrm{~mm}^{\circledR}$ ), câmera de vídeo $\left(\right.$ Toshiba $\left.^{\circledR}\right)$, vídeo cassete (Sony $\left.{ }^{\circledR}\right)$, monitor de vídeo, fonte de luz (Storz ${ }^{\circledR}$ ) e sistema para captação de imagem ("Laudo \& Imagem"); após utilização de vasoconstritor tópico nasal (cloridrato de nafazolina) e spray anestésico (neotutocaína a 5\%).

O ecocardiograma foi realizado no setor de Ecocardiografia do Hospital, com ecocardiógrafo modelo HP ImagePoint $\mathrm{HX}^{\circledR}$. Durante 0 ecocardiograma pôde-se avaliar a presença de sinais sugestivos de hipertensão pulmonar, 
através da estimativa da pressão sistólica da artéria pulmonar (refluxo tricuspídeo e/ ou regurgitação pulmonar). Pacientes portadores de hipertensão pulmonar evidenciada ao ecocardiograma pré-operatório foram submetidos a um novo exame no 70 pós-operatório. Todos os exames foram realizados pelo mesmo examinador.

Todos os pacientes foram submetidos à adenoamigdalectomia em decúbito dorsal horizontal, sob anestesia geral e intubação orotraqueal. A adenoidectomia foi realizada pela técnica convencional, com curetas de Beckman de três tamanhos diferentes. Utilizou-se a técnica de dissecção submucosa para a realização da amigdalectomia. Todos os pacientes foram operados pelo mesmo otorrinolaringologista.

Utilizou-se para análise estatística o cálculo do coeficiente de correlação entre duas variáveis.

\section{RESULTADOS}

Foram incluídos dez $(71,43 \%)$ pacientes do sexo feminino e $4(28,57 \%)$, do sexo masculino (Gráfico 1 ). A idade média dos pacientes foi de 6,78 anos, tendo 0 mais novo 2 anos e o mais velho, 14 anos. 0 ronco foi a queixa mais comumente observada, sendo relatada por todos os maiores responsáveis (100\%) e considerada de elevada gravidade por $64,28 \%$ destes. A respiração bucal $(92,86 \%)$ foi o segundo sintoma em freqüência observado durante 0 preenchimento do questionário. A pnéia e obstrução nasal foram relatados em dez pacientes $(71,43 \%)$. Os resultados obtidos através do questionário encontram-se na Tabela 1.

A análise do grau do tamanho da amídala palatina através do exame otorrinolaringológico demonstrou sete (50\%) crianças com hipertrofia amigdaliana grau IV; cinco (35,71\%), grau III e dois (14,29\%), grau II. Não foi observado nenhum paciente com obstrução menor que $25 \%$ ao nível da orofaringe.

Dos quatorze pacientes incluídos no estudo, doze $(85,71 \%)$ realizaram o $\mathrm{Rx}$ de cavum. Cinco pacientes $(41,67 \%)$ apresentaram obstrução adenoideana grau IV; seis (50\%), grau III e um paciente (8,33\%), grau II. Nenhum paciente apresentou hipertrofia adenoideana grau I, ou seja, obstrução menor que $25 \%$ da luz da rinofaringe.

Todos os 14 pacientes foram submetidos à avaliação do tamanho das vegetações adenoideanas por vídeonasofibroscopia. Nove $(64,28 \%)$ pacientes apresentaram obstrução proporcionada pela vegetação adenoideana maior que $75 \%$ da luz ao nível da rinofaringe; três $(21,43 \%)$, obstrução entre $50-75 \%$ da luz do órgão e dois $(14,29 \%)$ pacientes, obstrução entre $25-50 \%$ (Gráfico 2).

Um (7,14\%) paciente apresentou alteração ao exame ecocardiográfico, evidenciando-se sinais de hipertensão pulmonar. Foi evidenciado grau IV de obstrução para o paciente em questão, tanto para as amígdalas palatinas quanto as vegetações adenoideanas. Após sete dias do procedimen-

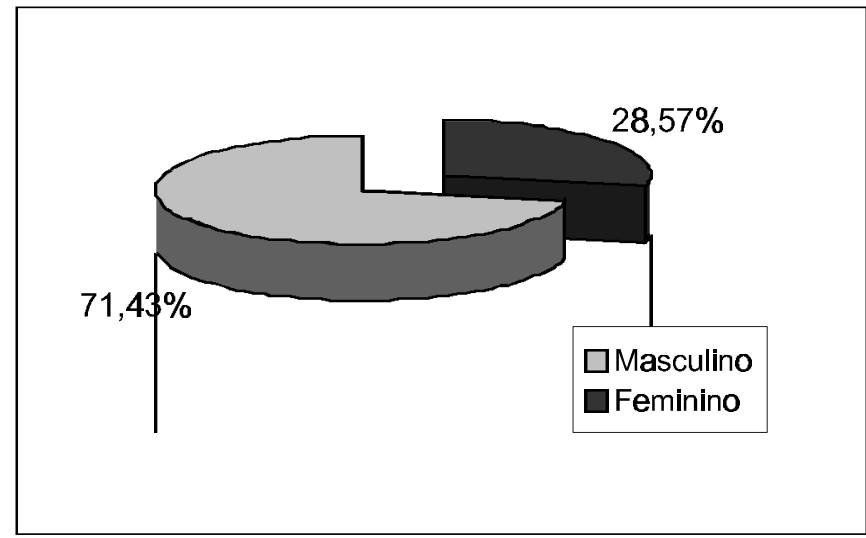

Gráfico 1. Distribuição dos pacientes conforme o sexo.

Tabela 1. Freqüência dos sintomas observados ao preenchimento do questionário pré-operatório por um maior responsável.

\begin{tabular}{lc}
\hline Queixa & N $(\%)$ \\
\hline Ronco & $14(100)$ \\
Respiração bucal & $13(71,43)$ \\
Obstrução nasal & $10(71,43)$ \\
Apnéia & $10(71,43)$ \\
Sono agitado & $09(64,29)$ \\
Halitose & $07(50)$ \\
Despertares noturnos & $05(35,71)$ \\
Hipersonolência diurna & $03(21,43)$ \\
Voz hiponasal & $03(21,43)$ \\
Enurese noturna & $02(14,29)$ \\
Pesadelos & $02(14,29)$ \\
Dificuldade de deglutição & $01(7,14)$ \\
\hline
\end{tabular}

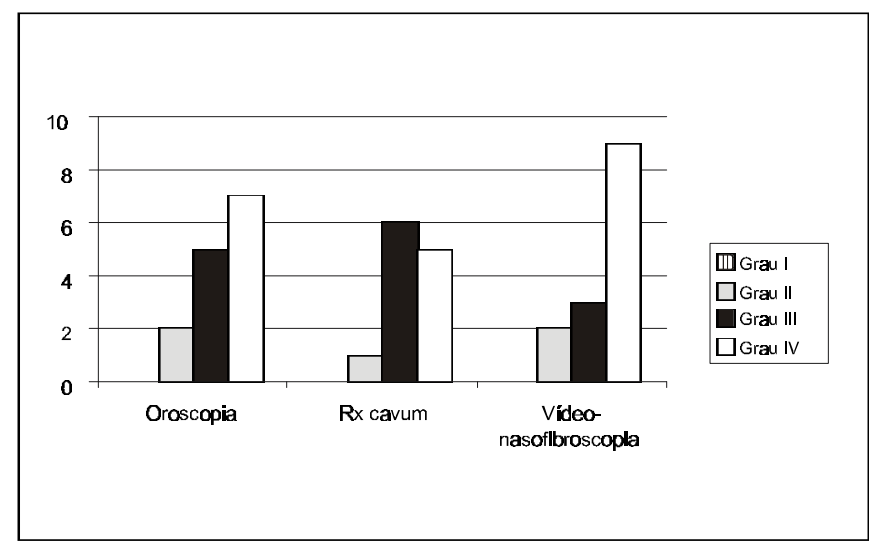

Gráfico 2. Distribuição dos pacientes conforme o grau de obstrução amigdaliana evidenciado à oroscopia e o grau de obstrução adenoideana observado ao Rx simples de cavum e à vídeonasofibroscopia. 
to cirúrgico, observou-se regressão dos sinais ecocardiográficos sugestivos de hipertensão pulmonar.

\section{DISCUSSÃO}

Apesar de a hipertrofia adenoamigdaliana ser uma condição clínica reconhecida desde os primórdios, foi somente descrita como causadora de hipertensão pulmonar e cor pulmonale em 1965 por Menashe e Farrehi ${ }^{2-5}$. Subsequentes autores (Noonan, 1965; Luke et al., 1966) também descreveram a hipertensão pulmonar originária do aumento excessivo das amígdalas palatinas e vegetações adenoideanas. Goodman creditou 0 atraso na documentação da hipertensão pulmonar decorrente da hipertrofia adenoamigdaliana a dois principais fatores: ao grande número de adenoamigdalectomias realizadas no passado e à incapacidade diagnóstica 6 .

A hipertrofia adenoamigdaliana é a principal causa de obstrução crônica de vias aéreas em crianças, principalmente naquelas entre 2-6 anos de idade, faixa etária caracterizada por aumento do tecido linfóide e subseqüente estreitamento da coluna aérea. A idade média de nossos pacientes foi de 6,78 anos, compatível com a faixa etária apresentada pela literatura de maior tamanho do tecido do anel linfático de Waldeyer.

Dentre toda a exuberante sintomatologia apresentada por crianças portadoras de hipertrofia adenoamigdaliana, o ronco é o grande motivador da procura por um especialista (otorrinolaringologista ou pediatra), destacando-se como a mais preocupante queixa apresentada pelos pais destas crianças ${ }^{6}$. Entretanto, outras queixas são observadas nestes pacientes, como: apnéia obstrutiva, hipersonolência diurna, enurese noturna, sono agitado, despertares noturnos, pobre desempenho escolar, diminuição das funções cognitivas (atenção e memória), pesadelos, irritabilidade, entre outros; inclusive a hipertensão pulmonar e o cor pulmonale. Em nosso estudo, o ronco $(100 \%)$ foi a queixa mais comumente observada entre os pais destas crianças. Porém não foi observado relação proporcional entre a gravidade relatada do sintoma e o grau de hipertrofia adenoamigdaliana. Respiração bucal, apnéia obstrutiva e obstrução nasal foram também queixas freqüentemente encontradas nestes pacientes com hipertrofia adenoamigdaliana.

Apesar da ocorrência de discordância entre as indicações e contra-indicações para a realização da adenoamigdalectomia, parece-nos bastante razoável a indicação deste procedimento cirúrgico para pacientes portadores de hipertrofia adenoamigdaliana, principalmente aqueles classificados como grau III ou IV. Em nosso estudo, doze $(85,71 \%)$ pacientes apresentavam hipertrofia amigdaliana grau III ou IV, não sendo evidenciado nenhum paciente com grau I de hipertrofia amigdaliana. De modo semelhante, nenhum paciente apresentou grau I de obstrução adenoideana evidenciada ao Rx de cavum ou à vídeonasofibroscopia. Houve correlação positiva entre o grau de obstrução amigdaliana evidenciado à oroscopia e o grau de hipertrofia adenoideana observado à vídeo-nasofibroscopia. Esta correlação positiva deve-se provavelmente ao crescimento uniforme e proporcional do tecido linfóide do anel de Waldeyer. O bservou-se também correlação entre a obstrução promovida pelas vegetações adenoideanas ao Rx de cavum e à vídeo-nasofibroscopia.

A fisiopatologia da hipertensão pulmonar desencadeada pela hipertrofia adenoamigdaliana encontra-se fundamentada na obstrução crônica das vias aéreas por um aumento exagerado da massa linfóide, promovendo um estreitamento da coluna aérea. Dessa forma, ocorre hipoventilação pulmonar e conseqüente retenção de $\mathrm{CO}_{2}$ (aumento da $\mathrm{P}_{\mathrm{a}} \mathrm{CO}_{2}$ ) e hipoxemia (diminuição da $\mathrm{P}_{\mathrm{a}} \mathrm{O}_{2}$ ). A retenção de $\mathrm{CO}_{2}$ acarreta bronco constrição pulmonar, aumentando o esforço respiratório. Paralelamente, a hipoxemia produz vasoconstrição pulmonar e, por conseguinte, aumento da pressão da artéria pulmonar, caracterizando a hipertensão pulmonar. Assim como já demonstrado por outros autores ${ }^{2-5}$, pudemos comprovar a hipertensão pulmonar com base fisiopatológica na hipertrofia adenoamigdaliana. Dessa forma, acreditamos que para pacientes com importante hipertrofia adenoamigdaliana e sintomatologia exuberante 0 ecocardiograma pode ser bastante útil na determinação da hipertensão pulmonar; especialmente por se tratar de um exame seguro, prático e não invasivo ${ }^{8}$.

A prevalência de hipertensão pulmonar em pacientes com hipertrofia adenoamigdaliana observada em nosso estudo foi de $7,14 \%$, sendo 0 quadro totalmente revertido após a adenoamigdalectomia, como evidenciado ao ecocardiograma pós-operatório. Dessa forma, acreditamos que a continuação deste estudo é de fundamental importância, pois apesar da pequena casuística, pudemos comprovar a existência desta condiç̧ão clínica em crianças atendidas em nosso Hospital.

\section{CONCLUSÃO}

Com base em nosso estudo, concluímos que a hipertrofia adenoamigdaliana também é causa de hipertensão pulmonar, sendo o ecocardiograma um exame bastante útil na determinação desta patologia, especialmente por se tratar de um exame seguro, prático e não invasivo.

Dessa forma, crianças em idade pré-escolar, apresentando sintomatologia obstrutiva exuberante (roncos, respiração bucal, apnéia e obstrução nasal) e hipertrofia adenoamigdaliana importante têm indicação potencial para adenoamigdalectomia, podendo reverter e eventualmente prevenir 0 aparecimento de hipertensão pulmonar decorrente do processo obstrutivo crônico desencadeado por aumento exagerado do tecido adenoamigdaliano. 


\section{REFERÊNCIASBIBUOGRÁFICAS}

1. Casselbrant M. Atualização nas indicações e contra-indicações das adenotonsilectomias. In: Sih T. Infectologia em Otorrinopediatria. Rio de Janeiro: Eds. Revinter; 2001. p. 63-7.

2. Capellari L, Guerra Jr G, Endo LH. Hipertrofia adenoamigdaliana e cor pulmonale. F méd (BR) 1990; 101(3):171-4.

3. Levin DL, Muster AL, Pachman LM, Wessel HU, Paul MH, Koshaba J. Cor pulmonale secondary to upper airway obstruction. Chest 1975; 68(2):166-70.

4. Ainger LE. Large tonsils and adenoids in small children with cor pulmonale. Brit Heart J 1968; 30:356-62.

5. Endo LH, Nicola EMD, Caldato MF. Cor pulmonale por hipertrofia de amígdalas e das adenóides - fatores predisponentes. Rev Bras Otorrinolaringologia $1981 ; 47: 83-90$.
6. Elsherif I, Kaesemullah C. Tonsil and adenoid surgery for upper airway obstruction in children. ENT J 1999; 78(8):617-20.

7. Goodman RS, Goodman M. Cardiac and pulmonary failure secondary to adenotonsillar hypertrophy. Laryngoscope 1976; 86(9):1367-74.

8. Miman MC, Kirazli T, Ozyurek R. Doppler echocardiography in adenotonsillar hypertrophy. Int J Pediatr Otorhinolaryngol 2000; 54(1):21-6.

9. Brodsky L, Koch J. Anatomic correlates ofnormal and diseased adenoids in children. Laryngoscope 1992; 102:1268-74.

10. Brouilette R, Hanson D, David R, Klemla L, Szatkowski A, Fembach S, Hunt C. A diagnostic approach to suspected obstructive sleep apnea in children. J Pediatr 1984; 105:10-4.

11. Piccirillo JF, Thawley SE. Sleep-disordered breathing. In: Cummings CW, Frederickson JM, Harker LA, Krause CJ, Richardson MA, Schuller DE, eds. St. Louis Missouri USA: Mosby-Year Book Inc; 1998. p. 1546-62. 